\title{
Diseño e implementación de prototipo de una pinza robótica adaptativa de tres dedos basada en estructura FinRay ${ }^{{ }^{*}}$
}

\author{
Carolina Silva** \\ José Gallardo***
}

Recibido: 23/10/2017 • Aceptado: 01/11/2018

https://doi.org/10.22395/rium.v18n34a7

\begin{abstract}
Resumen
Con el acelerado avance de la microelectrónica y los sistemas de procesamiento, la robótica está irrumpiendo constantemente en nuevas áreas además de la industria. Algunos manipuladores robóticos requieren maniobrar objetos con propiedades dimensionales variantes, superficies complejas y texturas delicadas, lo que lleva a la búsqueda de diseños de efectores finales que presenten nuevas características mecánicas y de control. Asimismo, con las investigaciones continuas que se han desarrollado en la disciplina científica de la biomímesis, una opción muy confiable y viable es replicar principios de la naturaleza para desarrollar aplicaciones tecnológicas. En este documento se describe el diseño mecánico y la implementación física de una pinza robótica adaptativa y subactuada con mordazas basadas en la estructura del efecto FinRay ${ }^{\circledR}$, que hace referencia a la reacción de movimiento de la aleta de un pez. Los resultados logrados demuestran su efectividad y adaptabilidad para la sujeción de distintos tipos de objetos
\end{abstract}

Palabras clave: pinza robótica; pinza adaptativa; estructura FinRay ${ }^{\circledR}$.

Artículo derivado de trabajo de grado de Maestría, Grupo de Investigación en Automatización y Robótica, Universidad Católica del Norte, Antofagasta, Chile.

** Ingeniera mecatrónica. Estudiante de Maestría en Ingeniería Informática, Universidad Católica del Norte, Av. Angamos 0610, Antofagasta, Chile, (+56) 55 2355157. Correo electrónico: carolina.silva02@alumnos.ucn.cl. Orcid: http://orcid.org/0000-0002-5863-1097

*** PhD. Académico, Doctor en Informática, Departamento de Ingeniería de Sistemas y Computación, Facultad de Ingeniería, Universidad Católica del Norte, Av. Angamos 0610, Antofagasta, Chile, (+56) 55 2355157. Correo electrónico: jgallardo@ucn.cl. Orcid: http://orcid.org/0000-0002-6477-5302 


\title{
Prototype design and implementing of a three-finger adaptive robotic gripper based on the FinRay ${ }^{\circledast}$ structure.
}

\begin{abstract}
Given the accelerated advancements in microelectronics and processing systems, robotics are constantly going forward in new areas alongside the industries. Some robotic handlers require being able to maneuver with objects with different dimensions, surfaces, and delicate textures, which sets the need for new final effectors with new mechanics and control features. Likewise, with the ongoing research developed in the bio-mimesis area, a very reliable and viable for replicating nature principles for the development of technological appliances. This document describes the mechanical design and the physical implementing process of an adaptive robotic gripper with jaws based on the FinRay ${ }^{\circledR}$ structure regarding the movement reaction of a fish fins. The achieved results show its effectivity and adaptability for the gripping of different objects.
\end{abstract}

Keywords: Robotic gripper; robotic grip; adaptive grip; FinRay ${ }^{\circledR}$ structure.

\section{Desenho e implantação de protótipo de uma pinça robótica adaptativa de três dedos baseada na estrutura FinRay ${ }^{\circledR}$}

\begin{abstract}
Resumo
Com o rápido avanço da microelectrónica e os sistemas de processamento, a robótica está irrompendo constantemente em novas áreas além da indústria. Alguns manipuladores robóticos requerem manobrar objetos com propriedades dimensionais variantes, superfícies complexas e texturas delicadas, o que leva à busca de desenhos de efeitos finais que apresentem novas características mecânicas e de controle. Além disso, com as pesquisas contínuas desenvolvidas na disciplina científica da biomímese, uma opção confiável e viável é reproduzir princípios da natureza para desenvolver aplicações tecnológicas. Neste documento, são é descrito o desenho mecânico e a implantação física de uma pinça robótica adaptativa e subatuada com mordaças baseadas na estrutura do efeito FinRay ${ }^{\circledR}$, que faz referência à reação de movimento da barbatana de um peixe. Os resultados demonstram sua efetividade e adaptabilidade para a fixação de diferentes objetos.
\end{abstract}

Palavras-chave: pinça robótica; pinça adaptativa; estrutura FinRay ${ }^{\circledR}$. 


\section{INTRODUCCIÓN}

Debido al gran avance de la tecnología en cuanto a los sistemas de control, a los sistemas mecánicos y electrónicos, y al procesamiento digital de imágenes, las aplicaciones de efectores finales robóticos (manipuladores) también se han extendido. En lo referente a la investigación, es posible encontrar el desarrollo de diversas aplicaciones, por ejemplo, los manipuladores para la recolección de frutas y hortalizas en la agricultura [1], o para la manipulación delicada de especies frágiles en el arrecife profundo o para la sujeción de objetos cotidianos con el fin de brindar apoyo a personas con movilidad reducida [2], entre muchas otras más. En síntesis, desarrollar manipuladores con una mayor adaptabilidad para el manejo, no solo de objetos con tamaños y formas geométricas definidas, sino también de objetos con dimensiones variantes, caras irregulares y texturas diversas [3], es uno de los nuevos retos para la tecnología pues se combina la detección de los objetos empleando sistemas de visión artificial o sensores precisos, y la estructura mecánica del manipulador que provea flexibilidad, adaptabilidad y sujeción blanda [4].

El diseño y desarrollo de manipuladores aún tiene grandes retos. Replicar la mano humana en todo su contexto, es decir, llegar a construir el complejo sistema mecánico logrando la perfecta movilización y percepción sensorial que indique instantáneamente la temperatura de un objeto, cuán lisa es su superficie e incluso, cuán firmemente debe ser capturado [5] es aún un desafío. Para abordar estos retos se están desarrollando un conjunto de opciones en la configuración de manipuladores adaptativos blandos, como por ejemplo: una pinza robótica universal, una membrana que por succión puede sostener diversos tipos de objetos [6], el OctopusGripper propuesta de Festo en base a la estructura de tentáculos, el Rubber Gripper [7], el efecto FinRay ${ }^{\circledR}$ basado en las aletas de un pez [8] y otros.

Adaptar este tipo de configuraciones blandas en los actuadores de los robots permitiría extender la capacidad de sostener objetos con diferentes dimensiones y estructuras (sin dañarlos) [8]. En la actualidad la tecnología 3D permite diseñar prototipos de las configuraciones mencionadas. En este documento se presenta el diseño de un manipulador con la estructura basada en el efecto FinRay ${ }^{\circledR}$, con el objetivo de manipular diversos objetos cotidianos con configuraciones dimensionales regulares e irregulares, incluso con características frágiles. En este contexto, en este artículo se reporta el diseño de las mordazas de una pinza robótica, también se presenta la simulación del modelo cinemático y la construcción del mismo utilizando la tecnología de impresión en 3D, sin focalizarse en el diseño sensorial y electrónico del dispositivo. 
El documento contiene las siguientes secciones: en la sección 1 describe la estructura del efecto FinRay ${ }^{\circledR}$; en la sección 2 detalla los objetos de prueba; en la sección 3 presenta el modelo matemático; en las secciones 4 a la 6 se presenta el diseño del prototipo robótico y su implementación física; en la sección 7 se muestra la evaluación y la discusión de los resultados logrados y, finalmente, se encuentran las conclusiones y propuestas de trabajo a futuro.

\section{ESTRUCTURA FINRAY}

Existe una disciplina científica enfocada en la comprensión y el uso de la naturaleza como modelo a replicar, adaptar e inspirar conceptos y diseños en la ciencia e ingeniería; esta disciplina es denominada biomímesis. Los científicos que se desenvuelven en esta ciencia exploran reglas, conceptos, mecanismos y principios de la biología con el objetivo de inspirar nuevas posibilidades de ingeniería; incluyendo la fabricación, el esbozo de mecanismos, la selección de materiales, la elaboración de procesos y el desarrollo de algoritmos [9].

Dentro del área de la robótica, diversas empresas han desarrollado tecnologías basadas en comportamientos de la naturaleza. Un ejemplo muy reconocido es el de la empresa alemana Festo, que presentó un asistente manipulador biónico (Bionic Handling Assitant) enfocado esencialmente en la interacción máquina-humano. El brazo de este manipulador se inspiró en el mecanismo de una trompa del elefante, mientras que los dedos del manipulador (de ahora en adelante, pinza) se inspiraron en la aleta de un pez. La biomímesis comprende dos líneas de acción: un proceso análogo y un proceso de abstracción. El primer proceso se enfoca en problemas que se presentan cotidianamente y busca una solución a estos en los comportamientos de la naturaleza para inspirarse en ellos y replicarlos. El segundo, observa y analiza comportamientos de la naturaleza y luego se encarga de encajar dicho principio en alguna necesidad o problema. En este último se presenta la estructura del efecto FinRay ${ }^{\circledR}$ [9].

El efecto Fin-Ray ${ }^{\circledR}$ inventado en 1997 por Leif Kniese y patentado por EvoLogics GmbH Berlín, describe una construcción flexible para transferir movimiento [10]. Este efecto es el resultado de un estudio sobre la capacidad locomotora de un pez que es proporcionada por la acción de la aleta de la cola. En la figura 1 se ilustra la fuerza que sobre la aleta se ejerce por medio del control en los puntos de reacción [11]. Específicamente, el efecto Fin-Ray ${ }^{\circledR}$ se representa por un triángulo isósceles, en el que uno de sus lados lo conforma una banda rígida que se une mediante los puntos de reacción a los otros dos lados del triángulo que están constituidos por bandas flexibles. En su interior existen bandas rígidas intermedias que conforman una estructura articulada, como se observa en la figura 2. 


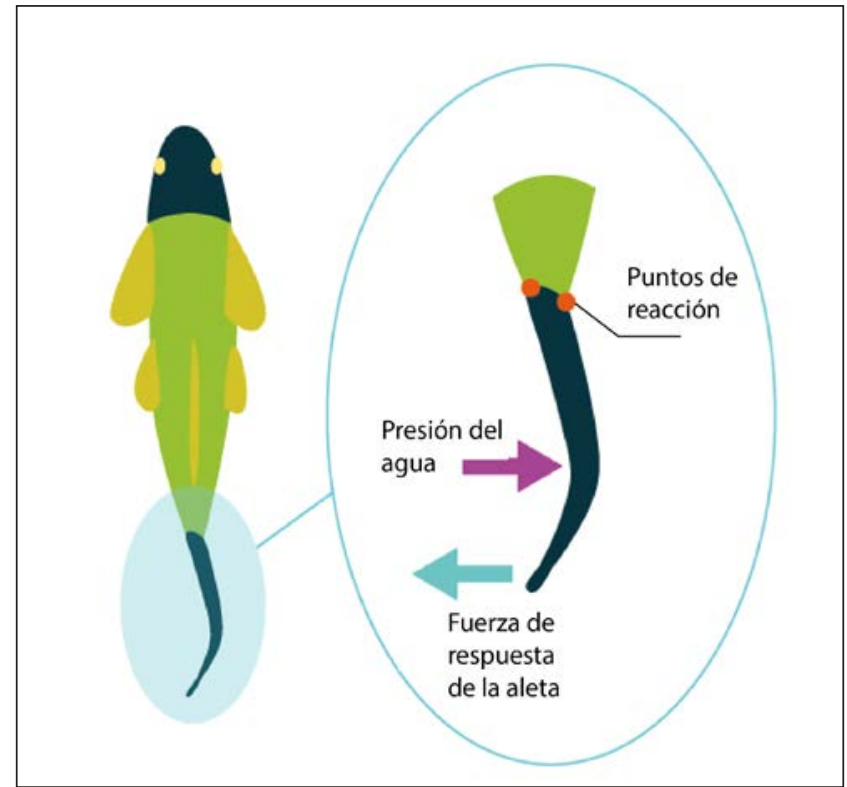

Figura 1. Estructura de la reacción de la aleta de un pez Fuente: elaboración propia.

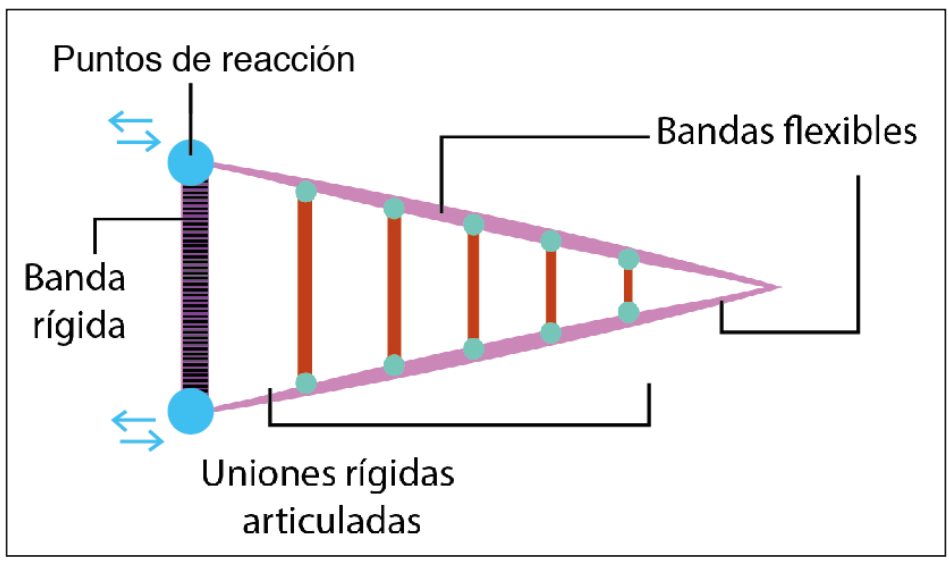

Figura 2. Estructura del efecto FinRay Fuente: elaboración propia.

Es necesario que este triángulo presente las subdivisiones con los elementos rígidos para crear trapezoides a lo largo de la estructura, de este modo se puede generar la presión individual en el momento de agarre de un objeto. En la figura 3 se presenta la mordaza sin los elementos rígidos intermedios y $\sin$ la estabilidad requerida cuando una fuerza externa ejerce acción en sus lados 
laterales. En contraste, la mordaza con bandas rígidas intermedias presenta mayor estabilidad (figura 4). Las barras intermedias ayudan a que la mordaza se acomode apropiadamente al objeto regular o irregular.

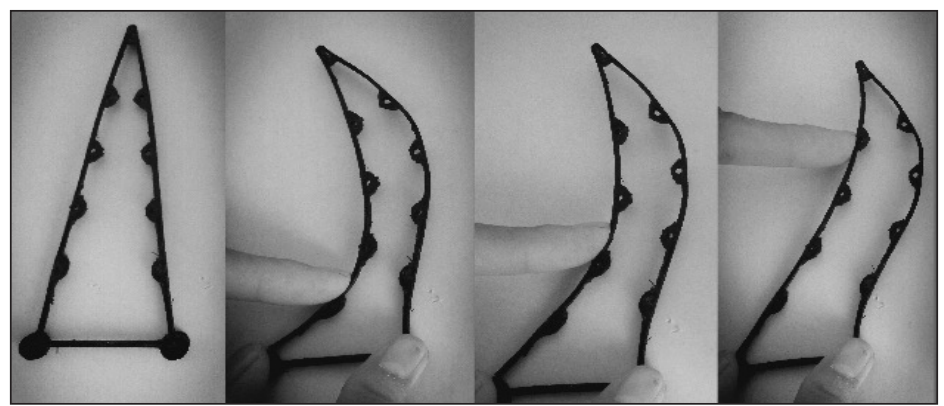

Figura 3. Estructura de la base triangular isósceles sin barras rígidas intermedias Fuente: elaboración propia.

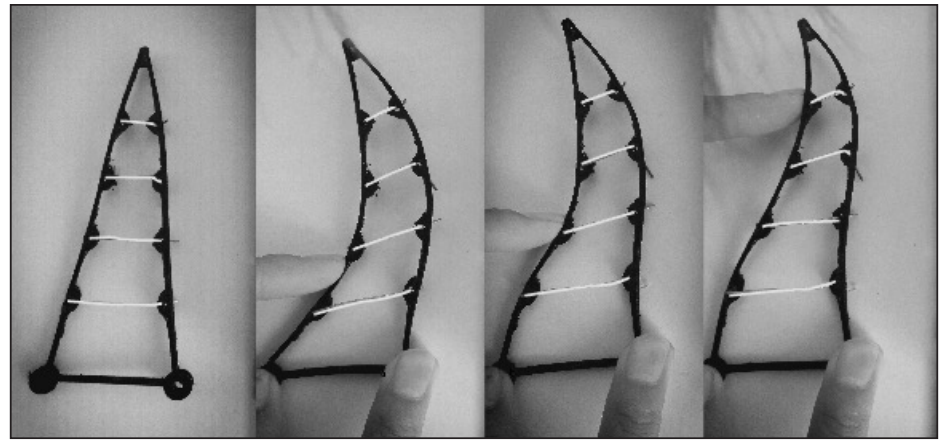

Figura 4. Estructura de la base triangular isósceles con barras rígidas intermedias Fuente: elaboración propia.

\section{OBJETOS DE PRUEBA A MANIPULAR}

Los objetos a ser manipulados están compuestos de diversos materiales (plástico, cerámica, cartón, goma). Tres de los objetos seleccionados para realizar las pruebas de presión sin dañarlos son: un tomate, un huevo y una bombilla (figura 5). Las dimensiones de los objetos varían entre un diámetro de 1,2 cm hasta los $13 \mathrm{~cm}$, y una altura máxima de $9 \mathrm{~cm}$, con un peso máximo de 700 gramos.

\section{MODELO CINEMÁTICO DE LAS MORDAZAS Y LA PINZA ADAPTATIVA}

El diseño matemático de la pinza es esencial para evaluar su movimiento espacial y conocer su área de trabajo, así como también, para evaluar si las dimensiones propuestas son suficientes para sostener los objetos de prueba. Con ese objetivo, se toma la posición 
y orientación que debe tener cada eslabón antes y durante el transcurso de la acción de manipulación del objeto.

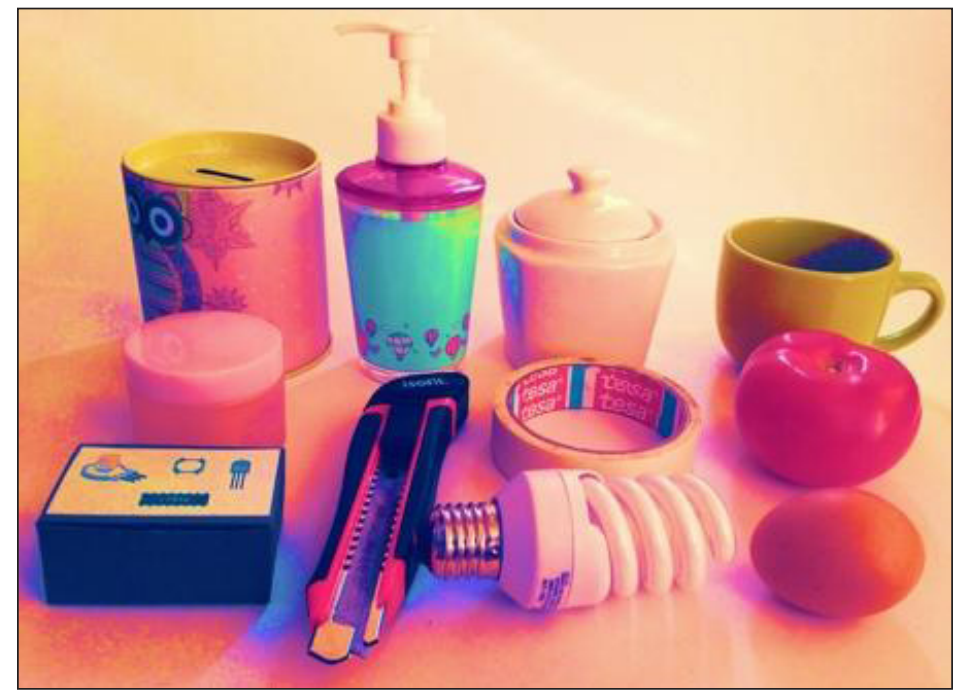

Figura 5. Objetos de experimentación para la manipulación de la pinza adaptativa Fuente: elaboración propia.

Para obtener la relación de movimiento entre los diferentes eslabones de la pinza, y controlar así la posición del efecto final, que en este caso se considera la yema de cada dedo, se deben obtener ciertas coordenadas mediante transformaciones que involucran la matriz de rotación. Estas matrices dependen directamente del eje sobre el cual está girando el robot, que para este caso sería la pinza. Las configuraciones de dichas matrices de los ejes $\mathrm{X}, \mathrm{Y}$ y $\mathrm{Z}$ con los ángulos de rotación $\alpha, \phi$ y $\theta$ respectivamente, se describen en las ecuaciones de la (1) a la (3) [12].

Para obtener la relación de movimiento entre los diferentes eslabones de la pinza, y controlar así la posición del efecto final, que en este caso se considera la yema de cada dedo, se deben obtener ciertas coordenadas mediante transformaciones que involucran la matriz de rotación. Estas matrices dependen directamente del eje sobre el cual está girando el robot, que para este caso sería la pinza. Las configuraciones de dichas matrices de los ejes $\mathrm{X}$, Y y Z con los ángulos de rotación $\alpha, \phi$ y $\theta$ respectivamente, se describen en las ecuaciones de la (1) a la (3) [12].

$$
R(x, \alpha)=\left[\begin{array}{ccc}
1 & 0 & 0 \\
0 & \cos \alpha & -\operatorname{sen} \alpha \\
0 & \operatorname{sen} \alpha & \cos \alpha
\end{array}\right]
$$




$$
\begin{aligned}
\mathrm{R}(\mathrm{y}, \phi) & =\left[\begin{array}{ccc}
\cos \phi & 0 & \operatorname{sen} \phi \\
0 & 1 & 0 \\
-\operatorname{sen} \phi & 0 & \cos \phi
\end{array}\right] \\
R(z, \theta) & =\left[\begin{array}{ccc}
\cos \theta & -\operatorname{sen} \theta & 0 \\
\operatorname{sen} \theta & \cos \theta & 0 \\
0 & 0 & 1
\end{array}\right]
\end{aligned}
$$

Con este, se considera el método matricial de Denavit-Hartenberg (D-H) para obtener la localización que debe tomar cada sistema de coordenadas $\{\mathrm{Si}\}$, ligado a cada eslabón i de una cadena articulada. Para ello, cada matriz de transformación ${ }^{i-1} A_{i}$ toma en cuenta cuatro transformaciones básicas que son:

1. $\operatorname{Rotz}\left(\theta_{i}\right)$ : rotación alrededor del eje $z_{i-1}$ un ángulo $\theta_{i}$.

2. $T\left(0,0, d_{i}\right)$ : traslación a lo largo de $z_{i-1}$ una distancia $d_{i}$; vector $d\left(0,0, d_{i}\right)$.

3. $T\left(a_{i}, 0,0\right)$ : traslación a lo largo de $x_{i}$ una distancia $a_{i}$; vector $a\left(a_{i}, 0,0\right)$.

4. $\operatorname{Rotx}\left(\alpha_{i}\right)$ :rotación alrededor del eje $x_{i}$ un ángulo $\alpha_{i}$.

Estas transformaciones consisten en una sucesión de traslaciones y rotaciones que permiten relacionar el sistema de referencia del elemento $i-1$ con el sistema del elemento $i$ [12], obteniéndose la ecuación (4).

$$
{ }^{i-1} A_{i}=\operatorname{Rotz}\left(\theta_{i}\right) T\left(0,0, d_{i}\right) T\left(a_{i}, 0,0\right) \operatorname{Rotx}\left(\alpha_{i}\right)
$$

Reemplazando por las matrices correspondientes, se obtendrá la ecuación (5):

$$
{ }^{i-1} A_{i}=\left[\begin{array}{cccc}
\cos \theta_{i} & -\cos \alpha_{i} \operatorname{sen} \theta_{i} & \operatorname{sen} \theta_{i} \operatorname{sen} \alpha_{i} & a_{i} \cos \theta_{i} \\
\operatorname{sen} \theta_{i} & \cos \alpha_{i} \cos \theta_{i} & -\cos \theta_{i} \operatorname{sen} \alpha_{i} & a_{i} \operatorname{sen} \theta_{i} \\
0 & \operatorname{sen} \alpha_{i} & \cos \alpha_{i} & d_{i} \\
0 & 0 & 0 & 1
\end{array}\right]
$$

Donde $\theta_{i}, a_{i}, d_{i}, \alpha_{i}$, son los parámetros Denavit-Hartenberg (D-H) del eslabón $i$.

Para obtener estos parámetros, primero se identifican las dimensiones y estructura de cada mordaza que conformará la pinza. Considerando los parámetros que se observan en la figura 6 , se elabora la tabla 1. 


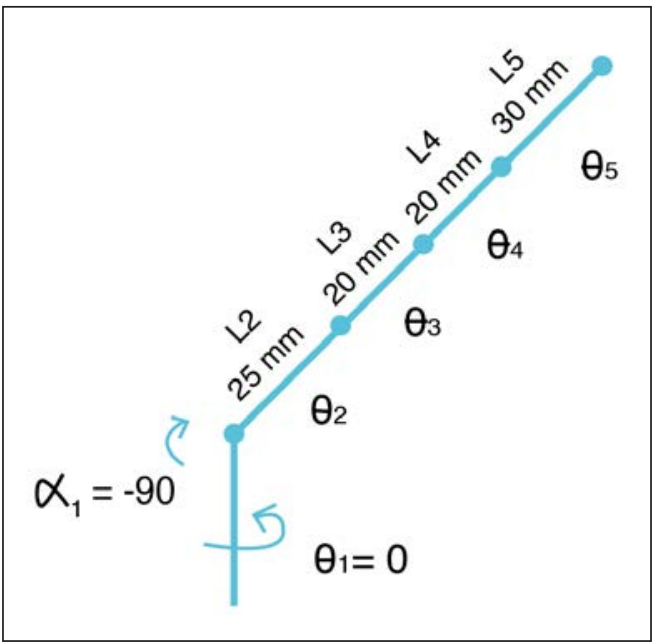

Figura 6. Identificación de los parámetros DH de la mordaza Fuente: elaboración propia.

Tabla 1. Parámetros D-H de la pinza adaptativa propuesta

\begin{tabular}{ccccc}
\hline Eslabón & $\theta_{1}$ & $d_{i}$ & $a_{i}$ & $\alpha_{i}$ \\
\hline 1 & $C$ & 0 & 0 & $-90^{\circ}$ \\
\hline 2 & $\theta_{2}$ & 0 & $l_{1}(2,5 \mathrm{~cm})$ & 0 \\
\hline 3 & $\theta_{3}$ & 0 & $l_{2}(2,0 \mathrm{~cm})$ & 0 \\
\hline 4 & $\theta_{4}$ & 0 & $l_{3}(2,0 \mathrm{~cm})$ & 0 \\
\hline 5 & $\theta_{5}$ & 0 & $l_{4}(3,0 \mathrm{~cm})$ & 0 \\
\hline \multicolumn{5}{c}{ Fuente: elaboración propia. }
\end{tabular}

Con los parámetros de la tabla 1 y aplicados a la matriz de la ecuación (5), se obtiene la matriz de transformación de cada uno de los elementos de la cadena cinemática de la ecuación (6).

Con los parámetros de la tabla 1 y aplicados a la matriz de la ecuación (5), se obtiene la matriz de transformación de cada uno de los elementos de la cadena cinemática de la ecuación (6).

$$
{ }^{0} A_{1}=\left[\begin{array}{cccc}
\cos \theta_{1} & -\operatorname{sen} \theta_{1} * \cos \alpha_{1} & \operatorname{sen} \theta_{1} * \operatorname{sen} \alpha_{1} & 0 \\
\operatorname{sen} \theta_{1} & \cos \theta_{1} * \cos \alpha_{1} & -\cos \theta_{1} * \operatorname{sen} \alpha_{1} & 0 \\
0 & \operatorname{sen} \alpha_{1} \cos \alpha_{1} & \cos \alpha_{1} & 0 \\
0 & 0 & 0 & 1
\end{array}\right]
$$




$$
\begin{aligned}
{ }^{1} A_{2} & =\left[\begin{array}{cccc}
\cos \theta_{2} & -\operatorname{sen} \theta_{2} & 0 & 25 * \cos \theta_{2} \\
\operatorname{sen} \theta_{2} & \cos \theta_{2} & 0 & 25 * \operatorname{sen} \theta_{2} \\
0 & 0 & 1 & 0 \\
0 & 0 & 0 & 1
\end{array}\right] \\
{ }^{2} A_{3} & =\left[\begin{array}{cccc}
\cos \theta_{3} & -\operatorname{sen} \theta_{3} & 0 & 20 * \cos \theta_{3} \\
\operatorname{sen} \theta_{3} & \cos \theta_{3} & 0 & 20 * \operatorname{sen} \theta_{3} \\
0 & 0 & 1 & 0 \\
0 & 0 & 0 & 1
\end{array}\right] \\
{ }^{3} A_{4} & =\left[\begin{array}{cccc}
\cos \theta_{4} & -\operatorname{sen} \theta_{4} & 0 & 20 * \cos \theta_{4} \\
\operatorname{sen} \theta_{4} & \cos \theta_{4} & 0 & 20 * \operatorname{sen} \theta_{4} \\
0 & 0 & 1 & 0 \\
0 & 0 & 0 & 1
\end{array}\right] \\
{ }^{4} A_{5} & =\left[\begin{array}{cccc}
\cos \theta_{5} & -\operatorname{sen} \theta_{5} & 0 & 30 * \cos \theta_{5} \\
\operatorname{sen} \theta_{5} & \cos \theta_{5} & 0 & 30 * \operatorname{sen} \theta_{5} \\
0 & 0 & 1 & 0 \\
0 & 0 & 0 & 1
\end{array}\right]
\end{aligned}
$$

La multiplicación final de estas matrices (ecuaciones 6 a 10) es la cinemática final de un dedo de la pinza robótica.

$$
{ }^{0} A_{5}={ }^{0} A_{1} *{ }^{1} A_{2} *{ }^{2} A_{3} *{ }^{3} A_{4} *{ }^{4} A_{5}
$$

Del resultado de esta operación (ecuación 11) se obtienen los valores para la posición final $\mathbf{X}, \mathbf{y}, \mathbf{z}$ de la yema del dedo, basado en los parámetros detallados en la figura 6, como se describe en las ecuaciones (12), (13) y (14).

$$
\begin{aligned}
x= & 30 * \cos \left(\theta_{2}+\theta_{3}+\theta_{4}+\theta_{5}\right)+20 * \cos \left(\theta_{2}+\theta_{3}+\theta_{4}\right)+ \\
& 20 * \cos \left(\theta_{2}+\theta_{3}\right)+25 * \cos \left(\theta_{2}\right) \\
y= & \left(\cos \left(\alpha_{1}\right)\right) * 30 * \operatorname{sen}\left(\theta_{2}+\theta_{3}+\theta_{4}+\theta_{5}\right)+20 * \\
& \quad \operatorname{sen}\left(\theta_{2}+\theta_{3}+\theta_{4}\right)+20 * \operatorname{sen}\left(\theta_{2}+\theta_{3}\right)+25 * \operatorname{sen}\left(\theta_{2}\right) \\
z= & \left(\operatorname{sen}\left(\alpha_{1}\right)\right) * 30 * \operatorname{sen}\left(\theta_{2}+\theta_{3}+\theta_{4}+\theta_{5}\right)+ \\
& 20 * \operatorname{sen}\left(\theta_{2}+\theta_{3}+\theta_{4}\right)+20 * \operatorname{sen}\left(\theta_{2}+\theta_{3}\right)+25 * \operatorname{sen}\left(\theta_{2}\right)
\end{aligned}
$$




\section{SIMULACIÓN DE LA PINZA ROBÓTICA EN MATLAB}

Con base en las ecuaciones de la 12 a la 14 y a la herramienta Robotics Toolbox de Matlab, se realiza de manera previa a la construcción física una simulación del comportamiento de la pinza adaptativa en posición inicial (figura 7) y cuando sostiene un objeto (figura 8) para evaluar su comportamiento [13]. A partir de dicha simulación, y después de observar el rango de apertura y el cierre para sostener un objeto, se encontró que las dimensiones propuestas son factibles para el diseño del prototipo de la pinza.

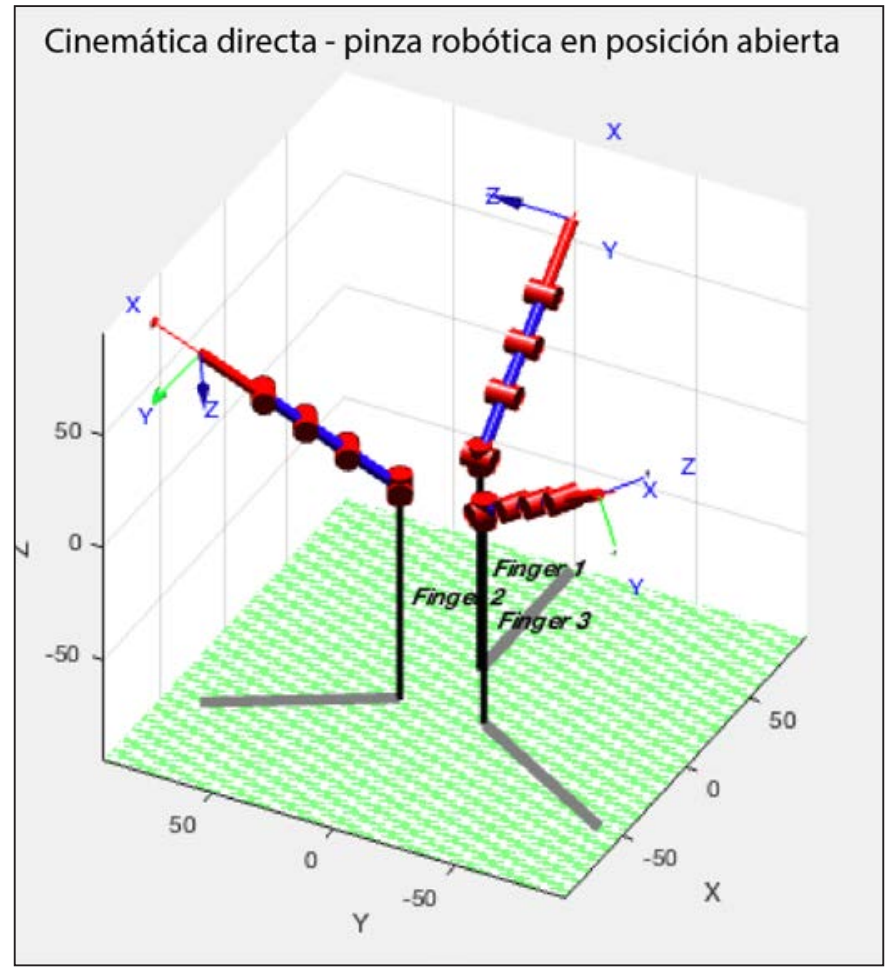

Figura 7. Simulación en Matlab de la pinza adaptativa en posición inicial Fuente: elaboración propia.

\section{DISEÑO E IMPLEMENTACIÓN DE LA PINZA ADAPTATIVA}

Para el accionamiento de un manipulador se emplean actuadores que pueden ser eléctricos, neumáticos o hidráulicos. El objetivo particular es mover una o más articulaciones de los dedos del manipulador. Las arquitecturas de manipulación que se presentan en el diseño de pinzas robóticas se dividen en tres categorías que se describen en la tabla 2, allí se consideran dos variables muy importantes: “N” que es el número de eslabones de la pinza y “M” que es el número de actuadores dentro de la misma [14, 15]. 


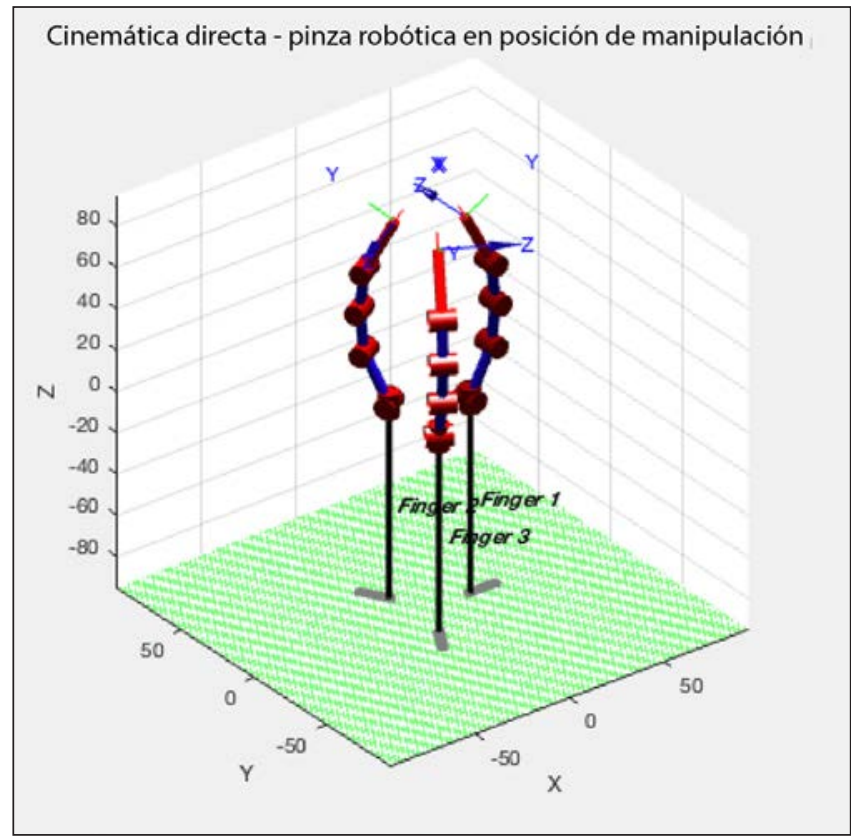

Figura 8. Simulación en Matlab de la pinza adaptativa sosteniendo un objeto Fuente: elaboración propia.

Tabla 2. Arquitecturas de manipulación

\begin{tabular}{cl}
\hline Relación entre $M y N$ & \multicolumn{1}{c}{ Características } \\
\hline $\mathrm{M}<\mathrm{N}$ & Algunos eslabones son considerados pasivos, acoplados o subactuados \\
\hline $\mathrm{M}=\mathrm{N}$ & $\begin{array}{l}\text { Cada eslabón tiene su propio actuador, no existen eslabones pasivos, } \\
\text { acoplados o subactuados. }\end{array}$ \\
\hline $\mathrm{M}>\mathrm{N}$ & Un eslabón usa más de un actuador para ser operado \\
\hline
\end{tabular}

Fuente: elaboración propia.

A partir de la información de la tabla 2, la pinza adaptativa presentada en este documento pertenece a una arquitectura subactuada debido a que se concentrará principalmente en el diseño del mecanismo interno que utiliza un solo actuador que puede accionar todos los dedos que hacen parte de la pinza robótica. La pinza robótica tendrá una estructura trípode, sus tres dedos o mordazas estarán distribuidas en una base circular a $120^{\circ}$ de separación una de la otra con el objetivo de manipular correctamente objetos con caras uniformes y deformes.

La pinza fue diseñada en el software CAD SolidWorks y creada mediante la tecnología de impresión en 3D con el material PLA (Polylactic Acid), que es un plástico 
biodegradable. Debido a ello, cada mordaza en su estructura triangular presenta tres uniones rígidas articuladas internas, característica que tiene la estructura del efecto FinRay ${ }^{\circledR}$ mostrada anteriormente en la figura 2. Sin embargo, es necesario mencionar que, si el material de la mordaza fuese más blando y flexible, se debería considerar dividir la mordaza con un mayor número de uniones rígidas articuladas internas, para compensar la presión que se ejercería al manipular los objetos. Además, el grosor de la pinza podría ser mayor. En este caso, por utilizar plástico PLA se optó por un grosor de $1.5 \mathrm{~mm}$.

Las dimensiones de cada mordaza se indican en la figura 9.a; en la figura 9.b se observa que la sección de la base y de la yema son más extensas que la sección intermedia. Esto se debe a que la primera sección tendrá una pequeña área muerta que se conecta tanto a la base de estructura triangular, así como también a los puntos de reacción. En cuanto a la sección de la yema, esta deberá ser más extensa que las otras por el hecho de que en ella es donde se ejerce la fuerza de reacción y la adaptación a los objetos a manipular.

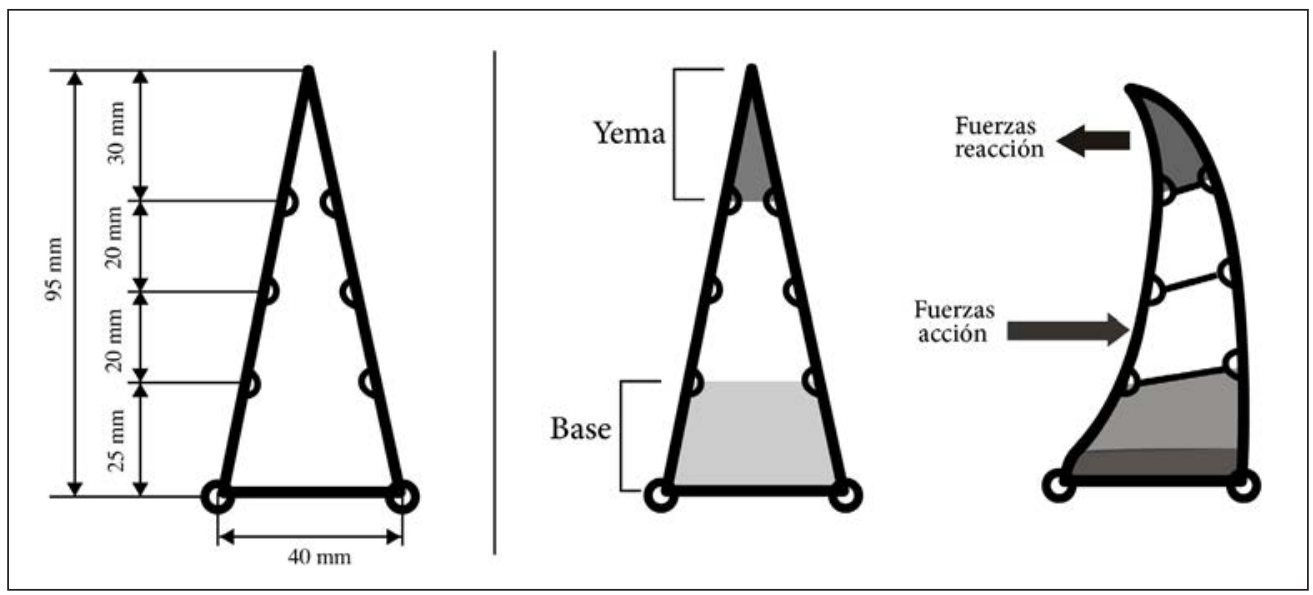

a)

b)

Figura 9. a) dimensiones de la mordaza; b) características de su estructura Fuente: elaboración propia.

Para el mecanismo de esta estructura, uno de los puntos de reacción debe estar sujeto a una base estable y rígida, mientras que el otro debe sujetarse a una base desplazable. Como se muestra en la figura 10, los sujetadores que se encuentran en los lados de la base cilíndrica exterior son rígidos y están sujetos a un punto de reacción de la mordaza, el otro punto de reacción está conectado al cilindro central (gris claro) que se puede desplazar en dirección vertical. 


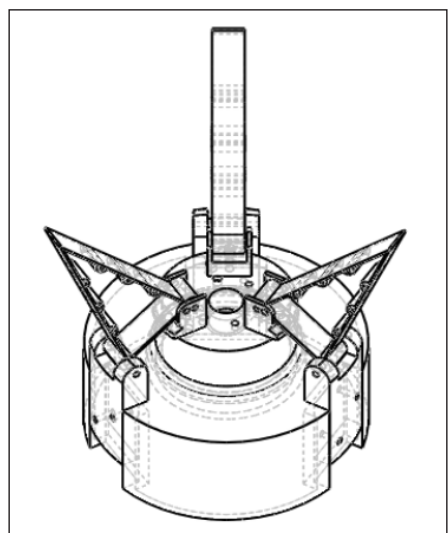

Figura 10. Posición de los puntos de acción de la mordaza en secciones rígidas y desplazables (el diseño de la pinza se realizó en SolidWorks). Fuente: elaboración propia.

Como se mencionó anteriormente, el diseño de esta pinza es subactuada. Por tanto, es el cilindro central el encargado de accionar el movimiento de las tres mordazas en la apertura y en el cierre. El rango de apertura o cierre de la pinza robótica depende en gran proporción de la distancia del desplazamiento vertical (figura 11). Para que la manipulación del objeto sea mucho mejor, cada mordaza posee en su lámina de presión una banda de púas de silicona (figura 12) con el objetivo de generar una fricción suave al momento de sostener cualquier objeto.

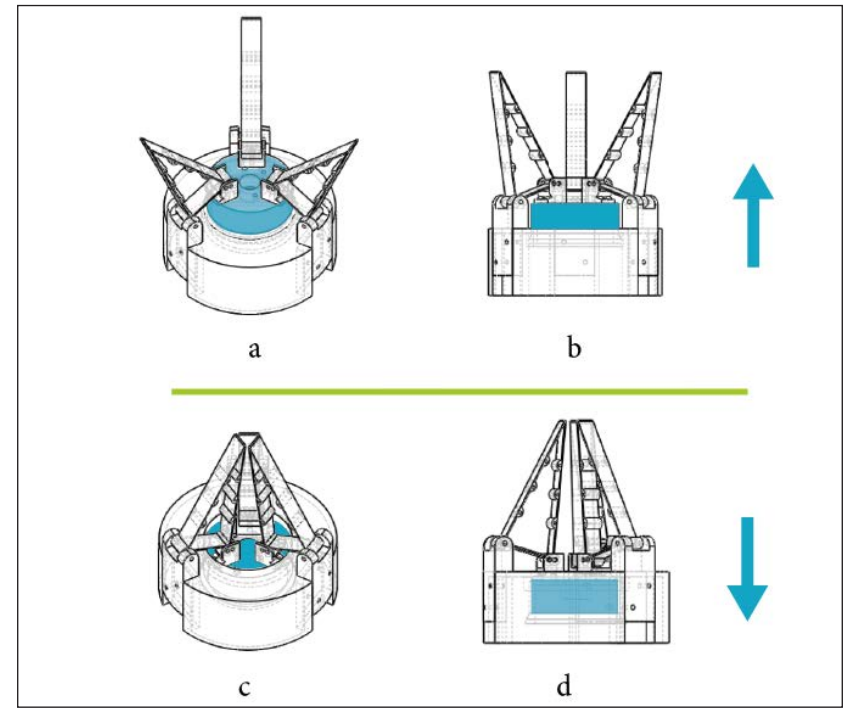

Figura 11. a) y b) corresponden a la vista frontal del mecanismo principal de la acción de apertura; c) y d) corresponden a la vista frontal del mecanismo de cierre. Fuente: elaboración propia. 


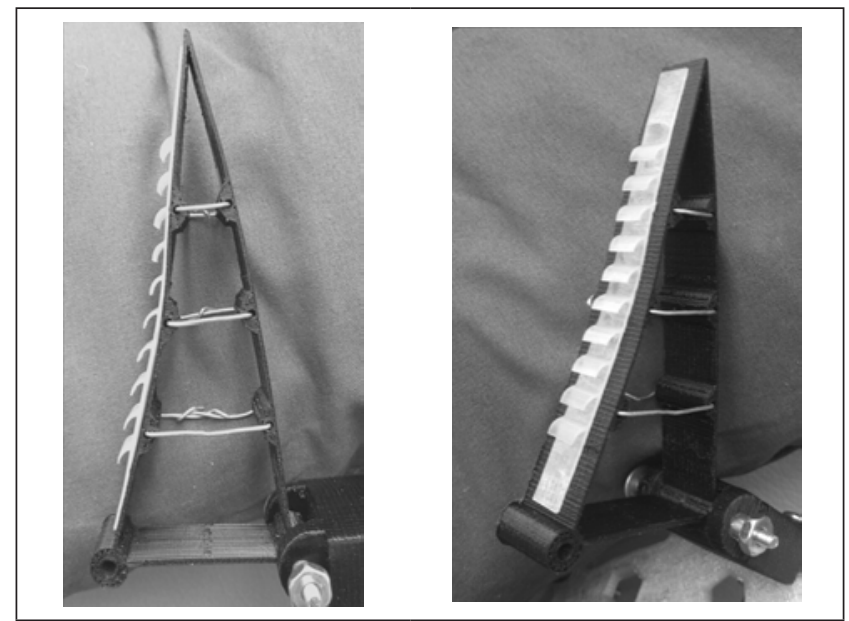

Figura 12. Púas de silicona para la lámina de presión de la mordaza Fuente: elaboración propia.

\section{EVALUACIÓN DE MANIPULACIÓN DE DIVERSOS OBJETOS}

Puesto que esta investigación se enfoca en el diseño de la pinza robótica basada en la estructura FinRay ${ }^{\circledR}$, la evaluación de la calidad de manipulación requiere de una previa acción manual de la pinza. Para el modelo de pinza desarrollado, se puede tener la apertura de las mordazas con un diámetro máximo de $13 \mathrm{~cm}$, y con capacidad para levantar objetos de hasta 780 gramos (debido a que el material de diseño es de plástico PLA). Sin embargo, debido a la estructura tipo trípode que presenta, se definen los límites de las dimensiones de los objetos a manipular (figura 13).

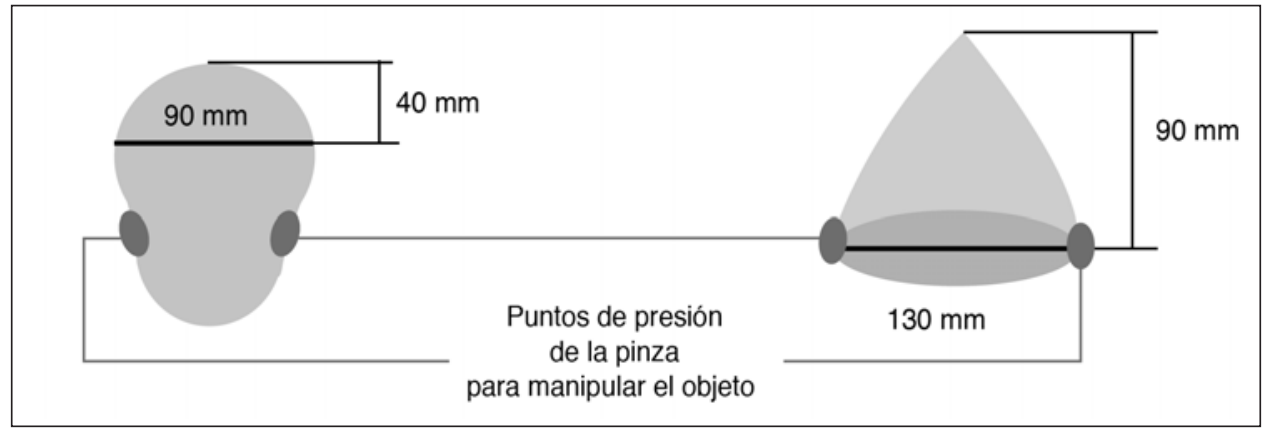

Figura 13. Dimensiones máximas que pueden poseer los objetos a manipular

Fuente: elaboración propia.

En la figura 14 a, b y c se muestra la manipulación de los objetos delicados mencionados anteriormente usando la pinza. Estos objetos no presentaron daño alguno en cuanto a la presión ejercida por la pinza robótica. 


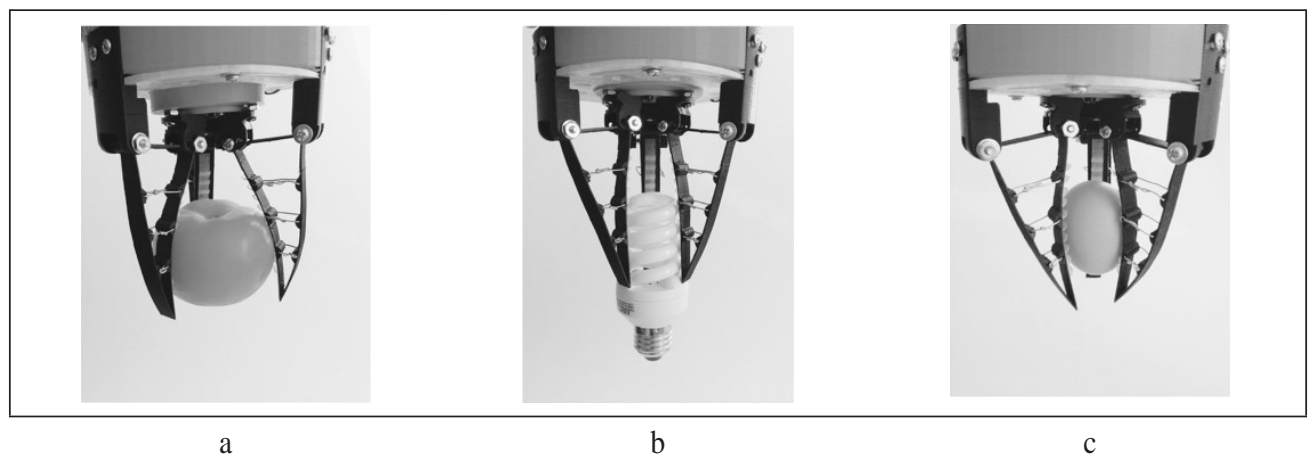

Figura 14. Objetos de textura delicada a) fruto tomate; b) bombilla; c) huevo.

Fuente: elaboración propia.

En la figura 15 a, b, c, d, e y f se muestra la manipulación de otros objetos de la prueba con materiales mixtos como porcelana, goma, plástico, metal y otros. Se puede observar que las mordazas se han adecuado a los objetos irregulares, por lo que se puede afirmar que la presencia de tres mordazas es suficiente para la manipulación de objetos de diferentes formas, materiales y tamaños. Puesto que las mordazas están distribuidas equitativamente en una base circular, el diámetro mínimo que se logró manipular es de 1,2 cm (figura 15d). Esta estructura mejora la capacidad para sostener objetos con caras curvas, planas o deformes si se compara con respecto a una pinza convencional de dos mordazas, esto es debido a que las tres mordazas se apoyan entre sí para distribuir correctamente la presión ejercida sobre objeto.

Además, la banda de púas de silicona que tiene cada mordaza favoreció la sujeción eficaz de objetos independientemente de la textura del material. Las subdivisiones en cada mordaza de la pinza permitieron la correcta adaptación a la forma de los objetos con texturas robustas y delicadas sin evidenciar deterioros sobre ellos. Esto último se registra en los objetos de prueba que se observan en las figuras 14 a, b, y c que son un tomate, una bombilla y un huevo, respectivamente.

\section{CONCLUSIONES}

El objetivo de esta investigación se focalizó en el diseño y la evaluación de la estructura de una pinza subactuada que es capaz de manipular una variedad de objetos con características de forma, tamaño y textura distintas. Fue posible manipular objetos desde un diámetro de $1,2 \mathrm{~cm}$ hasta $13 \mathrm{~cm}$, la altura máxima era de $9 \mathrm{~cm}$, y con un peso no mayor a 780 gramos.

El prototipo de pinza robótica fue producido mediante la tecnología de impresión 3D con material de plástico PLA. El grosor de cada mordaza es de $1.5 \mathrm{~mm}$ que 


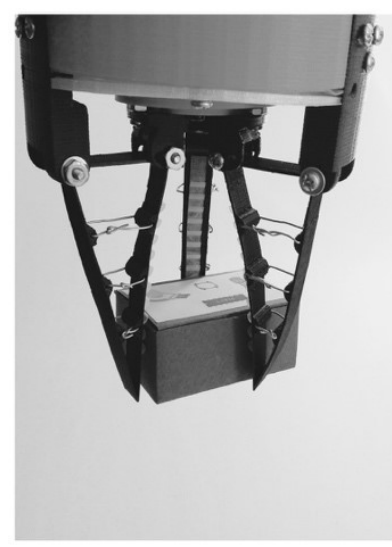

a.

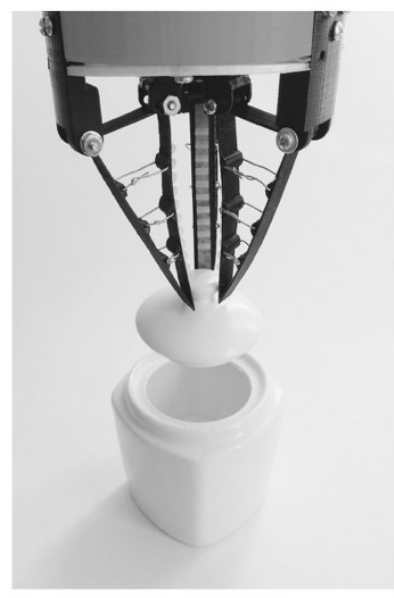

d.

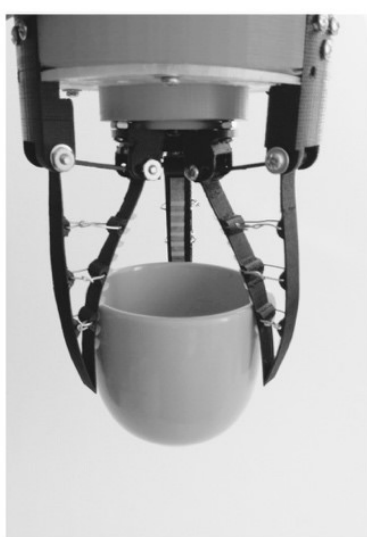

b.

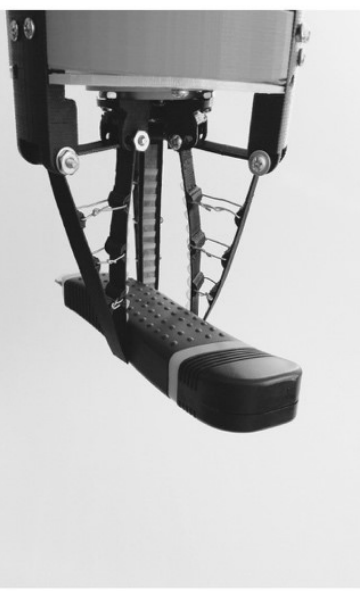

e.

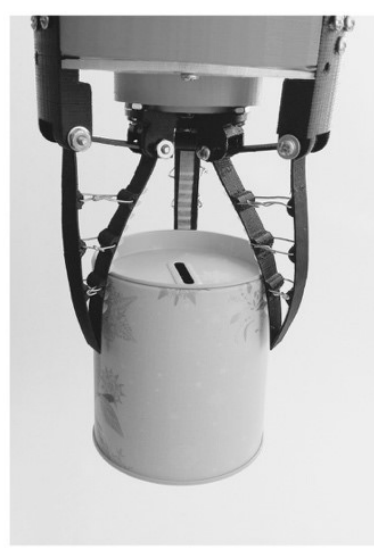

C.

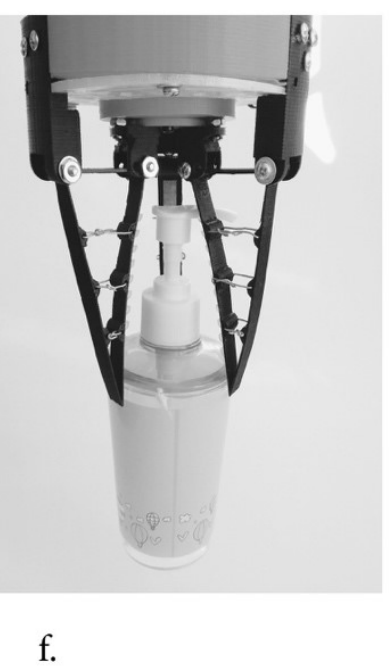

Figura 15. objetos de materiales diversos a) caja de cartón; b) taza de porcelana; c) envase metálico; d) tapa de azucarera de porcelana; e) cartonero con relieve de goma; f) envase de plástico. Fuente: elaboración propia.

permite generar cierta ductilidad en su estructura, puesto que se ha subdividido con barras rígidas en tres puntos dentro de la estructura triangular. Este diseño, más la incorporación de una banda de púas de silicona, ha permitido una manipulación mucho más eficiente independientemente del material que compone a los objetos de prueba. Sin la presencia de esta banda, la pared de agarre de cada mordaza sería lisa lo que provocaría deslizamientos de los objetos con facilidad.

La forma y peso de cada objeto de prueba hizo que se ejercieran fuerzas indirectas hacia las mordazas provocando así que ellas se adapten a la forma del objeto y que 
actúen con una presión correcta para su manipulación, como se observó, por ejemplo, al manipular un huevo y una taza de porcelana. La estructura trípode de la pinza genera una distribución eficiente de la presión al momento de manipular cualquier objeto en general. Sin embargo, existen problemas cuando los objetos son planos o muy delgados. Cuando se tienen cuerpos cúbicos se debe optar por otra configuración de mordazas diferente.

El desplazamiento vertical del cilindro central permite configurar el área de manipulación de la pinza robótica. Por tanto, es recomendable conocer la distancia vertical desplazada de las posiciones de apertura y cierre máximas que la pinza robótica posee física y mecánicamente en estado de reposo.

El prototipo de la pinza robótica que se presenta en este documento, evidencia la eficacia de este diseño para manipular objetos de características irregulares.

Como trabajo futuro de esta investigación, se automatizará el accionamiento de la pinza robótica diseñada considerando los torques necesarios respecto al volumen y el peso de los objetos a manipular. Además, de acompañarlo con un sistema de visión artificial para definir la apertura y cierre de las mordazas sobre el objeto.

\section{REFERENCIAS}

[1] J. Hemming, et al., "A robot for harvesting sweet-pepper in greenhouses". International Conference of Agricultural Engineering, Zúrich, 2014.

[2] W.Crooks, G. Vukasin, M. O’Sullivan, W. Messner, C. Rogers. "Fin Ray ${ }^{\circledR}$ Effect Inspired Soft Robotic Gripper: From the RoboSoft Grand Challenge toward Optimization” Frontiers in Robotics and AI, vol 3, pp. 70, 2016. DOI: 10.3389/frobt.2016.00070

[3] A. Zapciu, G. Constantin, D. Popescu, "Adaptive robotic end-effector with embedded 3Dprinted sensing circuits”, MATEC Web of Conferences, vol. 121, 2017.

[4] J. C. Yeo, H. K. Yap, W. Xi, Z. Wang , C.-H. Yeow, C. T. Lim. "Flexible and Stretchable Strain Sensing Actuator for Wearable Soft Robotic Applications”. Advanced Materials Technologies, vol 1, n. ${ }^{\circ}$ 3, pp. 1600018-1600026, May 2016

[5] R. D. Howe, A. M. Dollar, M. Claffee, "Robots get a Grip”, IEEE Spectrum, pp. 42-47, Dec. 2014.

[6] E. Brown, et al., "Universal robotic gripper based on the jamming of granular material", PNAS, vol 107, n. ${ }^{\circ}$ 44, pp 18809-18814, 2010.

[7] B. Homberg, R. Katzschmann, M.R. Dogar, D. Rus, "Haptic identification of objects using a modular soft robotic gripper”, IEEE/RSJ International Conference, Hamburg, Germany, 2015. 
[8] W. Crooks, S., Rozen-Levy, B. Trimmer, C. Rogers, W. Messner, "Passive gripper inspired by Manduca sexta and the Fin Ray ${ }^{\circledR}$ Effect.", International Journal of Advanced Robotic Systems, vol 14, n. ${ }^{\circ}$, 2016.

[9] Y. Bar-Cohen, “Biomimetics: Nature-Based Innovation”, Boca Ratón: CRC, 2016.

[10] O. Pfaff, S. Simeonov, I. Cirovic, P. Stano. "Application of FinRay ${ }^{\circledR}$ Effect approach for production process automation”, DAAAM International, vol 22, n. 1, pp. 1247-1248, 2011.

[11] W. Natchigall y N. Wisser, Bionics by Examples: 250 Scenaries from Classical to Modern Times. Alemania: Springer, pp 325, 2015.

[12] A. Barrientos, L. Peñin, C. Balaguer, R. Aracil, Fundamentos de Robótica, España: McGraw Hill, 2007.

[13] R. Miranda, Cinemática y Dinánima de Robots Manipuladores, México: Alfaomega, 2016.

[14] S. Khatib, Handbook of Robotics, Berlín: Springer, 2008.

[15] G. Kragten, Underactuated Hands. Fundamentals, performance analysis and design, $\mathrm{Ph}$. D, Technische Universiteit Delft, 2011. 